Pacific Journal of Mathematic 


\section{HOMOGENEOUS MODELS AND DECIDABILITY}

\section{TerRenCe Millar}

Fix a countable first order structure $\mathscr{A}$ realizing only recursive types. It is known that if $\mathscr{A}$ is prime or saturated then it is decidable iff the set of types it realizes is recursively enumerable. A natural conjecture was that the techniques of proof for those two cases could be combined to produce the result for those $\mathscr{A}$ that are homogeneous. This paper provides a negative answer to that conjecture.

For a complete decidable theory $T$, let $\left\{\theta_{i} \mid i<w\right\}$ be some fixed effective enumeration of all the formulas of $L(T)$. Then by an index for a recursive $n$-type $\Gamma\left(x_{1}, \cdots, x_{n}\right)$ of $T$ we mean a natural number $e$ satisfying:

$$
\{e\}(i)= \begin{cases}0 & \text { if } \theta_{i} \in \Gamma \\ 1 & \text { otherwise }\end{cases}
$$

(for notation, see [5]). Also, if $\Phi$ is a set of recursive types of $T$, then a witness set $A$ for $\Phi$ is a set of natural numbers satisfying:

(1) $\forall n \in A \exists \Gamma \in \Phi(n$ is an index for $\Gamma$ ); and

(2) $\forall \Gamma \in \Phi \exists n \in A$ ( $n$ is an index for $\Gamma$ ).

If $\Phi$ is exactly the set of types $\Gamma\left(x_{1}, \cdots, x_{n}\right)$ realized in some model $\mathscr{A}$ of $T$ satisfying $\left(x_{i} \neq x_{j}\right) \in \Gamma\left(x_{1}, \cdots, x_{n}\right), 1 \leqq i<j \leqq n, n<\omega$ then we also say that $A$ is a witness set for $\mathscr{A}$. Finally, a model $\mathscr{A}$ of $T$ is decidable just in case the theory of $\left(\mathscr{A}, a_{i}\right)_{i<\omega}$ is decidable for some indexing $\left\{a_{i} \mid i<\omega\right\}$ of $|\mathscr{A}|$. An undecidable model is a countable model that is not decidable.

Assume now that $\mathscr{C}$ is a prime model. Harrington [2] proved an equivalent version of (by the definitions, if a set of types has a witness set, then those types are recursive):

$\left(^{*}\right) \mathscr{C}$ is decidable iff $\mathscr{C}$ has an r.e. witness set.

From a recursion theoretic point of view, the principal device in the proof is a "wait and see" argument. Millar and Morley independently proved that $\left(^{*}\right)$ remains true when $\mathscr{C}$ is assumed to be countable and saturated. The principal recursion theoretic technique employed is a finite injury priority argument. Notice that a prime or saturated model is automatically homogeneous, and that any hemogeneous model is uniquely determined, up to isomorphism, by the set of types it realizes. It was therefore very natural that Morley asked whether $\left({ }^{*}\right)$ remained true under just the assumption that $\mathscr{C}$ was countable and homogeneous. This paper provides a negative answer. Interestingly, the construction exploits an "infinite injury". Sufficient con- 
ditions for such $\mathscr{C}$ to be decidable are discussed in, another one of interest is that $\left(^{*}\right)$ also holds if $\mathscr{C}$ is countable and recursively saturated:

THEOREM 1. If $\mathscr{A}$ is a countable, recursively saturated structure realizing only recursive types, then $\mathscr{A}$ is decidable if and only if $\mathscr{A}$ has a $\Sigma_{2}^{0}$ witness set.

Proof. In [Millar], the following theorem is proved.

Let $T$ be a complete decidable theory with a countable saturated model. Then $T$ has a decidable, homogeneous model realizing all of the recursive types of $T$ iff the set of recursive types of $T$ has a $\Sigma_{2}^{0}$ witness set.

In that paper, the only use made of the assumption that $T$ has a countable saturated model is in proving that every recursive, consistent (with $T$ ) set of formulas in a finite number of free variables is contained in a recursive type of $T$. Therefore, if we can show that $\mathrm{Th}(\mathscr{A})$ automatically has that derived property, then the conclusion of the theorem holds for $\mathrm{Th}(\mathscr{A})$. So let $\Phi\left(x_{1}, \cdots, x_{n}\right)$ be any recursive, consistent (with $\mathrm{Th}(\mathscr{A})$ ) set of formulas in the displayed free variables. Since $\mathscr{A}$ is recursively saturated, $\Phi$ is realized in $\mathscr{A}$ by some $\left\langle a_{1}, \cdots, a_{n}\right\rangle$. Then by the assumption that $\mathscr{C}$ realizes only recursive types, the type realized by $\left\langle a_{1}, \cdots, a_{n}\right\rangle$ is recursive and contains $\Phi$. Since obviously every recursive type of $\mathrm{Th}(\mathscr{A})$ is realized in $\mathscr{A}$, we see that $\mathrm{Th}(\mathscr{A})$ has a decidable homogeneous model $\mathscr{B}$ realizing every recursive type. Since recursively saturated structures are $\omega$-homogeneous, $\mathscr{A}$ and $\mathscr{B}$ are countable, homogeneous structures realizing exactly the same types. Therefore by a well known theorem, $\mathscr{A}$ and $\mathscr{B}$ are isomorphic. Thus is decidable.

THEOREM 2. There is a homogeneous undecidable model with an r.e. witness set.

In fact, we will specify a complete theory $T$ that has a countable homogeneous model $\mathscr{C}$ with an r.e. witness set $A$, such that no decidable model has $A$ as a witness set. Notice that if $\mathscr{B}$ is a decidable model and, $b_{1}, b_{2} \in|\mathscr{B}|$ realize the types $\Gamma_{1}, \Gamma_{2}$ respectively, then we can effectively find a recursive type $\Sigma$ realized in $\mathscr{B}$ such that $\Gamma_{1}\left(x_{1}\right) \cup \Gamma_{2}\left(x_{2}\right) \subset \Sigma\left(x_{1}, x_{2}\right)$. It is a slight variation on this ability that we "diagonalize across" in constructing $T . T$ will be the complete, model completion of a universal theory $T^{\prime}$. The language for $T$ contains unary predicated symbols $\left\{P_{i} \mid i<\omega\right\}$ and binary predicate 
symbols $\left\{S_{i} \mid i<\omega\right\}$. For recursive $F, G \subset 2^{<\omega}$ and recursive $H \subset 2^{<\omega} \times 2^{<\omega} \times 2^{<\omega}$ to be determined later, the axioms for $T^{\prime}$ are the universal closures of ( $2^{<\omega}$ is the set of finite sequences of 0 's and 1's):

( I ) $S_{i}(x, x)$ for all $i<\omega$;

(II) $S_{i}(x, y) \rightarrow S_{i}(y, x)$ for all $i<\omega$;

(III) $\neg \Lambda_{i<l(\underline{f})} P_{i}(x)^{\underline{f}(i)}$ for all $\underline{f} \in 2^{<\omega}-F$ (where $l(\underline{f})^{\prime}$ is the length of $f$ );

(IV) $\neg \Lambda_{i<l(\underline{g})} S_{i}(x, y)^{\underline{g}(i)}$ for all $g \in 2^{<\omega}-G$; and

(V) $]\left[\Lambda_{i<l\left(\underline{f_{0}}\right)} P_{i}(x)^{\underline{f}_{0}(i)} \wedge \Lambda_{i<l\left(\underline{f}_{1}\right)} P_{i}(y)^{\underline{f}_{1}(i)} \wedge \Lambda_{i<l(\underline{g})} S_{i}(x, y)^{\underline{g}(i)}\right] \quad$ for all $\left\langle\underline{f_{0}}, \underline{f}_{1}, \underline{g}\right\rangle \in H$,

where for any formula $\psi, \psi^{0} \equiv \psi$ and $\psi^{1} \equiv 7 \psi$. We must now determine $F, G$, and $H$.

Let $\left\{\mu_{n}: n<\omega\right\}$ be a fixed effective enumeration of all partial recursive $\mu: \omega \times \omega \times 2^{<\omega} \rightarrow 2$. For any partial recursive $v: \omega \times \omega \times$ $2^{<\omega} \rightarrow 2$, we denote by $v^{(s)}$ the function $\{\langle m, n, f, k\rangle \mid v(m, n, f)$ converges within $s$ steps and equals $k\}$. Notice that the domain and range of $v^{(s)}$ are recursive sets, uniformly in $s$. Also fix a total recursive $M: \omega \rightarrow \omega$ with the property that each element of $\omega$ has infinitely many pre-images and $M(n) \neq n$ for any $n<\omega . F_{n}, G_{n}$, and $H_{n}^{m}$ will be defined by an induction, and then we define $F=$ $\mathrm{U}_{n<\omega} F_{n}, G=\bigcup_{n<\omega} G_{n}$, and $H=\bigcup_{n, m<\omega} H_{n}^{m}$. We introduce various bookkeeping devices to facilitate the construction. First there will be markers $\left\{\square_{i}^{k} \mid k<3 ; i<\omega\right\}$ that will occasionally be defined on elements of $F$ and $G$. For each $n<\omega$, at most one element of $\omega$ will be $n$-fixed, whereas various elements might be $n$-designated (although never more than one at any one time). Also, each $n<\omega$ can be in one of three states: active, passive, or retired. We will use "proceed" in the construction to mean "go on to the next step of the induction". At the beginning of the construction, all markers are undefined and all states are active. At step $n, F_{n}, G_{n}$ and $H_{n}^{m}$ will be completely determined, $m<\omega$.

Step 0: $F_{0}=G_{0}=\{\langle 0\rangle,\langle 1\rangle\}, H_{0}^{m}=\varnothing, m<\omega$;

Step $n+1$ : There are two conditions that automatically put elements into the sets that we are defining:

(1) If $f \in F_{n}^{m}\left(G_{n}^{m}\right)$ then $F^{\wedge}\langle 0\rangle \in F_{n+1}^{m}\left(G_{n+1}^{m}\right)$; and

(2) $\underline{I}^{\wedge}\langle k, 1\rangle \in F_{n+1}, G_{n+1}$ for $k=0$ and 1 , where $\underline{I}$ is a sequence of $n-1$ zeros.

After this is done, then some of the markers are immediately defined or redefined according to: above;

(1) Define $\square_{n}^{k}$ on $\underline{I}^{\wedge}\langle 1, k\rangle$ in $F_{n+1}$ for $k=0,1$, where $\underline{I}$ is as

(2) Define $\square_{n}^{2}$ on $\underline{I}^{\wedge}\langle 1\rangle$ in $G_{n+1}$ for the same $I$; and 
(3) For each active $j<n$ and defined marker $\square_{j}^{i} i=0,1$, and $j \neq M(n)$ where let us say $\square_{j}^{i}$ is defined on $\underline{f}_{i j}$ :

(a) Just in case there is a $j$-fixed integer (and by assumption, $j$ is active), redefine $\square_{j}^{0}$ on $\underline{f}_{0 j}^{\prime} \underline{I}^{\prime}$ in $F_{n+1}$ where $\underline{I}^{\prime}$ is a sequence of zeroes such that $\underline{f}_{0 j} I^{\prime}$ has length $n+2$; and

(b) If there is presently a $j$-designated number, then redefine $\square_{j}^{1}$ on $\underline{f}_{1 j} I^{\prime \prime}$ in $F_{n+1}$ in a similar fashion.

It is easy to check that the required elements are in $F_{n+1}$ in the instructions for (a) and (b) above, we leave this to the reader.

For notational convenience, let $m$ be equal to $M(n)$. There are now several cases $\left(H_{n+1}^{i}=\varnothing\right.$ automatically for all $\left.i \neq m\right)$ :

( I ) If $\square_{m}^{0}$ is undefined or $m$ is retired, then put $H_{n+1}^{m}=\varnothing$ and proceed;

(II) If $\square_{m}^{0}$ is defined and $m$ is active, but there is presently no $m$-fixed integer, then let $\underline{f}$ be the element on which $\square_{m}^{0}$ is presently defined, and put $H_{n}^{m}=\varnothing$. If there is no $s<n$ such that $\mu_{m}^{(n)}(s, s, f)=0$, then proceed. Otherwise, $m$-fix the least such $s$, and then proceed;

(III) If $\square_{m}^{0}$ is defined, $m$ is active, there is an $m$-fixed integer, but there is no $m$-designated integer, then let $\underline{f}, \underline{g}$ be the elements on which $\square_{m}^{1}, \square_{m}^{2}$ are defined, respectively, let $m_{0}$ be the $m$-fixed integer. If there is no $s<n$ such that $\mu_{m}^{(n)}(s, s, \underline{f})=\mu_{m}^{(n)}\left(m_{0}, s, \underline{g}\right)=0$, then proceed. If such an $s$ exists that is $m_{0}$ or has been previously $m$-designated, then retire $m$ and proceed. Otherwise $m$-designate the least such $s$ and then proceed;

(IV) If $m$ is active but none of (I)-(III) apply, then let $g$ be the element on which $\square_{m}^{2}$ is defined, $m_{0}$ the $m$-fixed integer, and $d$ the $m$-designated integer.

(A) If $\mu_{m}^{(n)}\left(m_{0}, d, \underline{g^{\wedge}}\langle k\rangle\right) \neq 0$ for both $k=0$ and 1 , then put $H_{n+1}^{m}=\varnothing$ and proceed;

(B) If $\mu_{m}^{(n)}\left(m_{0}, d, \underline{g}^{\wedge}\langle k\rangle\right)=0$ for both $k=0$ and 1 , then retire $m$, put $H_{n+1}^{m}=\varnothing$ and proceed;

(C) If neither (A) nor (B), then let $\underline{f}_{i}$ be the element of $F_{n}$ (notice " $F_{n}$ ", not " $F_{n+1}$ ") that $\square_{m}^{i}$ was defined on $i=0,1$, and let $k$ be such that $\mu_{m}^{(n)}\left(m_{0}, d, \underline{g}^{\wedge}\langle k\rangle\right)=0$. Now, in this case

(i) $\hat{f}_{i}^{\wedge}\langle 1\rangle \in F_{n+1}$ for $i=0,1$;

(ii) $\left\langle\underline{f}_{0}^{\wedge}\langle 0\rangle, \underline{f}_{1}^{\wedge}\langle 0\rangle, \underline{g}^{\wedge}\langle k\rangle\right\rangle \in H_{n}^{m}$; and

(iii) $m$ is changed to the passive state;

after this is done, then proceed.

(V) If $m$ is passive, then put $H_{n+1}^{m}=\varnothing$ and let $m_{0}$ and $d$ be as above, and let $\left\langle\underline{f}_{0}^{\wedge}\langle 0\rangle, \underline{f}_{1}^{\wedge}\langle 0\rangle, \underline{g^{\wedge}}\langle k\rangle\right\rangle$ be the last element put into $\mathrm{U}_{i<n} H_{i}^{m}$ (again, that this exists is left to the reader to check). If either of $\mu_{m}^{(n)}\left(m_{0}, m_{0}, \underline{f}_{0}^{\wedge}\langle 1\rangle\right), \mu_{m}^{(n)}\left(d, d, \underline{f}_{1}^{\wedge}\langle 0\rangle\right)$ are undefined, then proceed. Otherwise, 
(A) If $\mu_{m}^{(n)}\left(m_{0}, m_{0}, \underline{f}_{0}^{\wedge}\langle 1\rangle\right)=1$ and $\mu_{m}^{(n)}\left(d, d, \underline{f}_{1}^{\wedge}\langle 0\rangle\right)=0$, then retire $m$ and proceed;

(B) If both values are 1, then:

(i) $\square_{m}^{i}$ is redefined on $\underline{f}_{\hat{i}}\langle i\rangle, i=0,1$;

(ii) $\square_{m}^{2}$ is redefined on $\underline{g}^{\wedge}\langle k\rangle^{\wedge} \underline{I}^{\prime}$ in $G_{n}$ (not $G_{n+1}$ ), where $\underline{I}^{\prime}$ is the appropriate sequence of zeros;

(iii) $\underline{g}^{\wedge}\langle k\rangle^{\wedge} \underline{I}^{\prime \wedge}\langle 1\rangle \in G_{n+1}, \underline{I}^{\prime}$ as in (ii); and

(iv) $m$ is changed to the active state, after this is done, then proceed.

(C) If $\mu_{m}^{(n)}\left(m_{0}, m_{0}, f_{0}^{\wedge}\langle 1\rangle\right)=0$ and not $(A)$, then:

(i) $m$ is changed to the active state;

(ii) $\square_{m}^{i}$ is redefined on $\underline{f}_{i}^{\wedge}\langle 1-i\rangle, i=0,1$;

(iii) $\square_{m}^{2}$ is redefined on $\underline{g}^{\wedge}\langle 1-k\rangle^{\wedge} \underline{I}^{\wedge}\langle 1\rangle \in G_{n}$ (not $G_{n+1}$ ), where $\underline{I}^{\prime}$ is the appropriate sequence of zeroes;

(iv) $\left\langle\underline{g^{\wedge}}\langle 1-k\rangle^{\wedge} \underline{I}^{\prime \wedge}\langle 1\rangle\right\rangle \in G_{n+1}$; and

(v) $d$ is no longer $m$-designated. This ends the construction.

Define the partial ordering $\leqq$ on $2^{<\omega} \cup 2^{\omega}$ by $f \leqq g$ just in case $\underline{f}$ is a proper initial segment of $g$, or $\underline{f}=\underline{g}$. Next we list some of the important properties of our end products:

Lemma 1. (i ) $F=\bigcup_{n<\omega} F_{n}, G=\bigcup_{n<\omega} G_{n}, H^{m}=\bigcup_{n<\omega} H_{n}^{m}$, and $H=\bigcup_{n<\omega} H^{m}$ are all recursive;

(ii) If $\underline{f} \in F(G)$ then $\underline{f}^{\wedge} \underline{I} \in F(G)$ for all finite sequences of zeros $I$;

(iii) Every element $\left\langle\underline{f}_{1}, \underline{f}_{2}, \underline{g}\right\rangle \in H$ satisfies $l\left(\underline{f}_{1}\right)=l\left(\underline{f}_{2}\right)$ and $\underline{f}_{1}(r)=$ $\underline{f}_{2}(r)=0$, where $r=l\left(\underline{f}_{1}\right)$;

(iv) If $\left\langle\underline{f}_{1}^{\wedge}\langle 0\rangle, \underline{f}_{2}^{\wedge}\langle 0\rangle, \underline{g^{\wedge}}\langle k\rangle\right\rangle \in H \quad$ then $\quad \underline{f}_{i}^{\wedge}\langle 1\rangle \in F, \quad i=1,2$, $g^{\wedge}\langle 1-k\rangle \in G$ and $g$ was marked by a $\square_{s}^{2}$ marked for some s;

( v) If $\left\langle\underline{f}_{2}^{\wedge}\langle 0\rangle, \underline{f}_{2}^{\wedge}\langle 0\rangle, g\right\rangle,\left\langle\underline{f}_{1}^{\prime}, \underline{f}_{2}^{\prime}, \underline{g}^{\prime}\right\rangle \in H^{m}$ and $l(\underline{g})<l\left(\underline{g}^{\prime}\right)$ then $\underline{f}_{i} \leqq \underline{f}_{i}^{\prime}$ for $i=1,2$ and $\underline{f}_{1}^{\wedge}\langle 0\rangle \leqq \underline{f}_{1}^{\prime}$ iff $\underline{f}_{2}^{\wedge}\langle 1\rangle \leqq \underline{f}_{2}^{\prime}$ iff $g \leqq g^{\prime} ;$ and

(vi) There are only countably many $f \in 2^{\omega}$ such that $f \in F(G)$ for all $\underline{f} \leqq f$.

Proof. The proof amounts to a routine check of the construction, and we leave most of the details to the reader. (ii) is a consequence of (1) at the beginning of step $n+1$. The second part of (iii) follows essentially from (IV) (C) (ii), since that constitues the only circumstance in which an element is placed in $H$. (iv) follows from the last remark and (IV)(C) (i) (iii), and (B) (iii). For (v) first notice by the above that when $\left\langle\underline{f}_{1}^{\wedge}\langle 0\rangle, \underline{f}_{2}^{\wedge}\langle 0\rangle, g\right\rangle$ is placed in $H^{m}$ during the construction, by (IV)(C) (iii) $m$ is then changed to the passive state. If another element is to appear in $H^{m}$ after that point, $m$ must return to the active state. This can only occur via (V), (B) or 
(C). Thus, that ( $\mathrm{v}$ ) holds follows simply from an examination of the instructions in those two cases and a simple induction. Finally, for (vi) we see that if $g \in 2^{\omega}$ is such that for infinitely many $g \leqq g$ there is an $f$ as in (vi) satisfying $g \leqq f$ and $f \neq g$, then for some $m$ and $i$, infinitely many $g \leqq g$ have $\square_{m}^{i}$ defined on them at the end of some stage of the construction. (vi) is straightforward now with the observation that if $f, \underline{g}$ are two elements on which a particular $\square_{m}^{i}$ is defined at the end of different stages, then one is an initial segment of the other. Next, we complete the list of axioms for $T$. Arbitrarily fix $0<r<\omega, \quad 0 \leqq n<\omega$ and a maximal subset $A\left(x_{1}, \cdots, x_{n}\right)$ of

$$
\left\{P_{i}\left(x_{j}\right)^{t}, S_{i}\left(x_{j}, x_{k}\right)^{t} \mid t=0,1 ; 1 \leqq j, k \leqq n ; i<r\right\}
$$

consistent with the set of those axioms of $T^{\prime}$ in which only predicate symbols with indices less than $r$ occur. It is easy to check that this can be done effectively, uniformly in $r$ and $n$. For the same $r$ and any $m>0$, let $B\left(x_{1}, \cdots, x_{n+m}\right)$ be another such set satisfying $A\left(x_{1}, \cdots, x_{n}\right) \subset B\left(x_{1}, \cdots, x_{n+m}\right)$. Then for all such $r, n, m, A$, and $B$ we include as an axiom for $T$ :

$$
\forall x_{1} \cdots \forall x_{n} \exists x_{n+1} \cdots \exists x_{n+m}\left[\Lambda A\left(x_{1}, \cdots, x_{n}\right) \longrightarrow \Lambda B\left(x_{1}, \cdots, x_{n+m}\right)\right] .
$$

The fact that there always is at least one such $B$ for every $A$ follows from the form of the axioms for $T^{\prime}$ and Lemma 1 (ii)-(iv). We will now outline a proof that $T$ is consistent. By compactness it is enough to show that for arbitrary such $A_{0}, B_{0}, \cdots, A_{s-1}, B_{s-1}$ as above,

$$
T^{\prime} \cup\left\{\forall x_{1} \cdots \forall x_{n_{i}} \exists x_{n_{i}+1} \cdots \exists x_{n_{i}+m_{i}}\left(\Lambda A_{i} \longrightarrow \Lambda B_{i}\right) \mid i<s\right\}
$$

is consistent. Assume that such a collection is fixed and we define a model $\mathscr{A}$ of $\left(^{*}\right)$, with universe a subset $\left\{a_{i} \mid i<\omega\right\}$, by specifying its diagram $\Delta_{\mathscr{A}}$ by an induction.

Step 0:

$$
P_{i}\left(a_{0}\right), S_{i}\left(a_{0}, a_{0}\right) \in \Delta_{\mathscr{A}} \text { for all } i<\omega .
$$

Assume inductively that membership in $\Delta_{\mathscr{A}}$ has been determined for exactly $P_{i}\left(a_{j}\right)^{t}, S_{i}\left(a_{j}, a_{k}\right)^{t} t=0,1 ; j, k<r$; and $i<\omega$, and is such that $\Delta_{\mathscr{A}}$ is consistent with $T^{\prime}$.

Step $r+1$ : Suppose $r=i[\bmod s]$. Then with respect to some enumeration of $\omega$ that we assume has been fixed before the beginning of the construction, let $\left\langle k_{1}, \cdots, k_{n_{i}}\right\rangle$ be the least element such that

$\exists x_{n_{i}+1} \cdots \exists x_{n_{i}+m}\left(\Lambda A\left(a_{k_{1}}, \cdots, a_{k_{n_{i}}}\right) \longrightarrow \Lambda B\left(a_{k_{1}}, \cdots, a_{k_{n_{i}}}, x_{n+1}, \cdots, x_{n_{i}+m_{i}}\right)\right]$

fails with respect to $\Delta_{\mathscr{A}}$, as presently determined. If there is no such element, then go on to the next stage. Otherwise we specify 
first that $B\left(a_{k_{1}}, \cdots, a_{k_{n_{i}}}, a_{r}, \cdots, a_{r^{+} m_{i}-1}\right) \subset \Delta_{\mathscr{A}}$. Next, we complete the description with respect to the new constant symbols, by another induction. Assume that membership of $P_{i}\left(a_{j}\right)^{t}, S_{i}\left(a_{j}, a_{k}\right)^{t}$ has heen determined for all $j, k<r+m_{i}$ and $i<v$. Fix $j<r+m_{i}$. Put $\neg P_{v}\left(a_{j}\right)$ into $\Delta$, unless $P_{v}\left(a_{j}\right)$ is already in $\Delta$, or $f^{\wedge}\langle 1\rangle \notin F$, where $f \in 2^{v}$ is such that $P_{i}\left(a_{j}\right)^{f(i)} \in \Delta$. for $i<v$. In either of the two alternative cases, simply ensure that $P_{v}\left(a_{j}\right)$ is in $\Delta \ldots$. Now fix in addition a $k<r+m_{i}$. Put $S_{v}\left(a_{j}, a_{k}\right)$ into $\Delta_{\mathscr{A}}$ unless its negation already belongs, in which case do nothing. This is done for each $j$ and $k$. This ends both inductions. We leave it to the reader to check via Lemma 1 that $\Delta$, can be expanded to the diagram of a model $\mathscr{A}$ of (*), pointing out that the axioms in $V$ are never endangered because of our attempt to put $\neg P_{i}\left(a_{j}\right)$ into $\Delta$, whenever "possible", the choice of the $A_{i}, B_{i}$ 's, and Lemma 1(iii)-(iv). The conclusion is that $T$ is consistent.

An obvious modification of the last argument shows that in fact every model of $T^{\prime}$ can be extended to a model of $T$. Next we claim that $T$ admits elimination of quantifiers. An equivalent condition is that for all models $\mathscr{A}, \mathscr{B}$ of $T$ and substructures $\mathscr{C} \subset \mathscr{A}, \mathscr{B}$, if an existential sentence with parameters from $|\mathscr{C}|$ holds in $\mathscr{A}$, then it holds in $\mathscr{B}$. So arbitrarily fix such $\mathscr{A}, \mathscr{B}, \mathscr{C}$ and $\exists y_{1} \cdots \exists y_{m} \theta\left(\underline{c}_{1}, \cdots, \underline{c}_{n}\right.$, $\left.y_{1}, \cdots, y_{m}\right)$ (where $\theta\left(x_{1}, \cdots, x_{n}, y_{1}, \cdots, y_{m}\right)$ is a quantifier free formula of $L(t))$. Therefore there are $\underline{a}_{1}, \cdots, \underline{a}_{n} \in|\mathscr{A}|$ such that

$$
\langle\mathscr{A}, \underline{\bar{c}}, \underline{\bar{a}}\rangle \vDash \theta(\bar{c}, \bar{a}) \text {. }
$$

Fix such $\underline{\bar{a}}$ and let $r$ be the least number greater than zero and all of the indices of predicate symbols that occur in $\theta$. Let $A\left(c_{1}, \cdots, c_{n}\right)$, $B\left(c_{1}, \cdots, c_{n}, a_{1}, \cdots, a_{m}\right)$ be the diagram of $\mathscr{C}^{\prime}$, $\mathscr{A}^{\prime \prime}$ respectively, where $\mathscr{A}^{\prime}$ is the reduct of the substructure of $\mathscr{A}$ with universe $\left\{\underline{c}_{i} \mid 1 \leqq i \leqq n\right\}$ to the language with only the predicate symbols whose indices are less than $r$, and similarly for $\mathscr{f}^{\prime \prime}$ (omitting equality). Since $\mathscr{A}$ is obviously also a model of $T^{\prime}$, an axiom for $T$ is:

$$
\forall x_{1} \cdots \forall x_{n} \exists x_{n+1} \cdots \exists x_{n+m}\left[\Lambda A\left(x_{1}, \cdots, x_{n}\right) \rightarrow \Lambda B\left(x_{1}, \cdots, x_{n+m}\right)\right] .
$$

Therefore, since

$$
(\mathscr{B}, \underline{\bar{c}}) \vDash \Lambda A(\bar{c}) .
$$

The conclusion follows.

Now let $\psi$ be an arbitrary sentence in $L(T)$ consistent with $T$. Let $\mathscr{A}$ be a model of $\{\psi\} \cup T$ and $\mathscr{B}$ an arbitrary model of $T$. By elimination of quantifiers there is a quantifier free $\theta(y)$ equivalent under $T$ to $(y=y \wedge \psi)$. If we simply repeat the last argument for $\mathscr{A}, \mathscr{B}, \theta$ and $\mathscr{C}=\varnothing$, we see that $T$ is complete. Since $T$ is 
axiomitizable it is also decidable. We will now specify the desired r.e. witness set.

By the elimination of quantifiers, to uniquely determine an $n$ type $\Gamma\left(x_{1}, \cdots, x_{n}\right)$ of $T$, it is sufficient to determine the maximal subset of

$$
\left\{P_{i}\left(x_{j}\right)^{t}, S_{i}\left(x_{j}, x_{k}\right)^{t},\left(x_{j}=x_{k}\right)^{t} \mid t=0,1 ; 1 \leqq j, k \leqq n ; i<\omega\right\}
$$

contained in $\Gamma\left(x_{1}, \cdots, x_{n}\right)$. Therefore, by the axioms in I and II and the decidability of $T$, an index for a recursive $\Gamma\left(x_{1}, \cdots, x_{n}\right)$ can effectively be obtained from indices for the (recursive) functions

$$
\left\{f_{i}, g_{j k} \in 2^{\omega}, h \in 2^{n \times n} \mid 1 \leqq j<k \leqq n ; 1 \leqq i \leqq n\right\},
$$

where

$$
P_{s}\left(x_{i}\right)^{f_{i}(s)}, S_{s}\left(x_{j}, x_{k}\right)^{g_{i k}(s)},\left(x_{j}=x_{k}\right)^{h(j-1, k-1)} \in \Gamma\left(x_{1}, \cdots, x_{n}\right)
$$

for the obvious values. Let $\mu: \omega \rightarrow B$ be a recursive, onto function, where

$$
\begin{aligned}
B= & \left\{\left\langle\underline{f}_{1}, \cdots, \underline{f}_{n}, \underline{g}_{12}, \cdots, \underline{g}_{1 n}, \underline{g}_{23}, \underline{g}_{23}, \cdots, g_{(n-1) n}\right\rangle \mid \underline{f}, \underline{g}^{\prime} s \in 2^{<\omega},\right. \\
& \text { all lengths equal; } 0<n<\omega\} .
\end{aligned}
$$

We will now effectively specify, uniformly in $s$, a type $\Gamma_{s}$ corresponding to $\mu(s)$.

Fix an $s<\omega$ and let

$$
\mu(s)=\left\langle\underline{f}_{1}, \cdots, \underline{f}_{n}, \underline{g}_{12}, \cdots, \underline{g}_{(n-1) n}\right\rangle,
$$

where the lengths are say $m$ (all of which of course can be uniformly effectively determined). Check first that:

(i) $\underline{f}_{i} \in F$ for $1 \leqq i \leqq n$;

(ii) $\underline{g}_{j k} \in G$ for $1 \leqq j<k \leqq n$; and

(iii) for each $j, k, 1 \leqq j<k \leqq n$ there is no $\left\langle\underline{f}_{1}^{\prime}, \underline{f}_{2}^{\prime}, \underline{g}^{\prime}\right\rangle \in H$, $l\left(\underline{f}_{1}^{\prime}\right) \leqq m$ such that $\underline{f}_{1}^{\prime} \leqq \underline{f}_{j}, \underline{f}_{2}^{\prime} \leqq \underline{f}_{k}$ and $\underline{g}^{\prime} \leqq \underline{g}_{j k}$.

If any of these fail, then the type $\Gamma\left(x_{1}, \cdots, x_{n}\right)$ will be the one containing

$$
\left\{P_{i}\left(x_{j}\right), S_{i}\left(x_{j}, x_{k}\right),\left(x_{j} \neq x_{k}\right) \mid i<\omega ; 1 \leqq j, k \leqq n\right\},
$$

Otherwise we define the required $f_{i}, g_{j k} \in 2^{\omega}, 1 \leqq i \leqq n, 1 \leqq j<k \leqq n$ by an induction.

Step 0: $f_{i}(r)=\underline{f}_{i}(r)$ and $g_{j k}(r)=g_{j k}(r)$ for all $r<m, 1 \leqq i \leqq n$, and $1 \leqq j<k \leqq n$. So assume their values have been determined for all arguments less than $p$, such that (i)-(iii) hold for the extensions.

Step $p^{\prime}:$ First of all $g_{j k}(p)=0$ for all $1 \leqq j<k \leqq n$. Let $f_{i}^{\prime}$ be $f_{i}$ as determined so far. Now check (iii) again with respect to $\left\langle f_{i}^{\prime \wedge}\langle 0\rangle, f_{k}^{\prime \wedge}\langle 0\rangle, g_{j k}^{\prime}\right\rangle$, for $1 \leqq j<k \leqq n$. For any $j, k$ for which (iii) 
fails, define $f_{j}(p)=f_{k}(p)=1$. After this is done define $f_{i}(p)=0$ for any $f_{i}(p)$ not yet defined. That the induction hypotheses are maintained can be checked via Lemma 1(ii)-(iv). This ends the induction. $\Gamma_{s}$ is now defined to be the unique type determined by its containment of

$$
\left\{P_{t}\left(x_{i}\right)^{f_{i}(t)}, S_{t}\left(x_{j}, x_{k}\right)^{g_{j k}(t)}, x_{j} \neq x_{k} \mid t<\omega ; 1 \leqq i \leqq n ; 1 \leqq j<k \leqq n\right\} .
$$

Lemma 2. $\left\{\Gamma_{s} \mid s<\omega\right\}$ has an r.e. witness set.

Proof. Straightforward. Let $\left\{\hat{\Gamma}_{s} \mid s<\omega\right\}$ be an r.e. witness set for $\left\{\Gamma_{s} \mid s<\omega\right\}$, where $\hat{\Gamma}_{s}$ is an index for $\Gamma_{s}$.

LEMMA 3. For all $s<\omega$ there is an $m$ such that no negated atomic formula containing a predicate symbol with index greater than $m$ belongs to $\Gamma_{s}$.

Proof. For the $S_{t}$ predicate symbols this is immediate, since the instructions specify that $g_{i j}(t)=0$ for all $t$ greater than the length of the corresponding $g_{i j}$. Notice next that since the $f_{j}(p)$ and $f_{k}(p)$ are defined to be 1 whenever (iii) fails with respect to $\left\langle\underline{f}_{j}^{\prime \wedge} 0, \underline{f}_{k}^{\prime \wedge} 0, \underline{g}_{j k}^{\prime}\right\rangle$, it follows by Lemma 1 (v) that (iii) can fail at most once for each such pair $j, k$. The lemma now follows by the instructions in the induction for what to do when (iii) does not fail. Call such types as in the lemma eventually zero (e.z.) types.

Lemma 4. $\left\{\hat{\Gamma}_{s} \mid s<\omega\right\}$ is a witness set for a countable model of $T$.

Proof. By a standard argument, $T \cup\left\{\Gamma_{s}\left(a_{1}^{s}, \cdots, a_{n_{s}}^{s}\right) \mid s<\omega\right\} \equiv T^{*}$ is a consistent theory, where the $a_{j}^{i}$ 's are distinct new constant symbols. We first claim that this theory has a countable model that omits every type of $T$ that is not e.z. By elimination of quantifiers for $T$ and Lemma 1 (vi), $T$ has only a countable number of types altogether. Therefore by the Omitting Types Theorem [1] and elimination of quantifiers it is sufficient to show that for every 2-type $\Gamma\left(x_{1}, x_{2}\right)$ of $T$ that is not e.z., and every formula $\theta\left(b_{1}, \cdots, b_{n}, x_{1}, x_{2}\right)$ (where the $b_{i}$ 's are $a_{k}^{j}$ 's), if $\exists x_{1} \exists x_{2} \theta\left(b_{1}, \cdots, b_{n}, x_{1}, x_{2}\right)$ is consistent with $T^{*}$, then so is $\exists x_{1} \exists x_{2}\left[\theta\left(b_{1}, \cdots, b_{n}, x_{1}, x_{2}\right) \wedge \neg \gamma\left(x_{1}, x_{2}\right)\right]$ for some $\gamma\left(x_{1}, x_{2}\right) \in \Gamma\left(x_{1}, x_{2}\right)$. So fix such a $\Gamma\left(x_{1}, x_{2}\right)$ and $\theta$, and we will find a $\gamma\left(x_{1}, x_{2}\right)$. Let $\psi$ be the quantifier free formula such that

$$
T \vdash\left[\theta\left(b_{1}, \cdots, b_{n}, x_{1}, x_{2}\right) \leftrightarrow \psi\left(b_{1}, \cdots, b_{n}, x_{1}, x_{2}\right)\right] .
$$

Let $m$ be larger than any index of any predicate symbol occurring in $\psi$. By the assumption of consistency there are $\underline{f}_{i}$ and $g_{j k}$ of 
length $m$ such that the following is consistent:

$$
\begin{aligned}
T^{*} \cup\left\{\psi\left(b_{1}, \cdots, b_{n+2}\right)\right\} \cup & \left\{P_{t}\left(b_{i}\right)^{f_{i}(t)}, S_{t}\left(b_{j}, b_{k}\right)^{g_{j k}(t)} \mid t<m ;\right. \\
& 1 \leqq i \leqq n+2 ; 1 \leqq j<k \leqq n+2\},
\end{aligned}
$$

where $b_{n+1}, b_{n+2}$ are new constant symbols. Now for the noneffective step. Essentially by Lemma 1 (iv)-(v), there is an $m^{\prime}>m$ and $\underline{f}_{i}^{\prime}, \underline{g}_{j k}^{\prime}$ such that for all $i, j, k, 1 \leqq i \leqq n+2,1 \leqq j<k \leqq n+2$ :

(a) $\underline{f}_{i} \leqq \underline{f}_{i}^{\prime}$ and $\underline{g}_{j k} \leqq \underline{g}_{j k}^{\prime}$;

(b) $\underline{f}_{i}^{\prime} \in \bar{F}$ and $\underline{g}_{j k}^{\prime} \in G$;

(c) if $S_{0}\left(b_{j}, b_{k}\right)^{g_{j k}(0)} \notin T^{*}$, then no marker $\square_{s}^{2}$ is ever defined on a $\underline{g}, \underline{g}_{j k} \not \leq \underline{g} \leqq \underline{g}_{j k}^{\prime}$ or $\underline{g}_{j k}^{\prime} \leqq \underline{g}$;

(d) if $S_{0}\left(b_{j}, b_{k}\right)^{\underline{g} j k(0)} \notin T^{*}$ and a $\square_{s}^{2}$ marker is defined on $g_{j k}$ at stage $m^{\prime}$, then it is defined there at all later stages;

(e) if $S_{0}\left(b_{j}, b_{k}\right)^{g_{j k}(0)} \in T^{*}$, then $S_{t}\left(b_{j}, b_{k}\right) \in T^{*}$ for all $t \geqq m^{\prime}$;

(f) $P_{t}\left(b_{i}\right) \in T^{*}$ for all $i, \Gamma, 1 \leqq i \leqq n, t \geqq m^{\prime}$;

(g) (茾) remains consistent when $m, \underline{f}_{i}, \underline{g}_{j k}$ are replaced by $m^{\prime}$, $\underline{f}_{i}^{\prime}, \underline{g}_{j k}^{\prime}$ respectivly.

Notice for (e) and (f) that all of the $\Gamma_{s}$ are e.z. types. Now let $f_{i}, g_{j k} \in 2^{\omega}$ be functions satisfying:

(i) $\underline{f}_{i}^{\prime} \leqq f_{i}, \underline{g}_{j k}^{\prime} \leqq g_{j k}$; and

(ii) $f_{i}(t)=g_{j k}(t)=0 ; 1 \leqq i \leqq n+2,1 \leqq j<k \leqq n+2 m^{\prime} \leqq t<\omega$. It follows that

$$
\left(^{+}\right) \quad\left\{P_{t}\left(b_{i}\right)^{f_{i(t)}}, S_{t}\left(b_{j}, b_{k}\right)^{g_{j k}(t)} \mid 1 \leqq i \leqq n+2 ; 1 \leqq j<k \leqq n+2 t<\omega\right\}
$$

is consistent with $T^{*}$. We leave the details for the reader to check, noting that the axioms of $T^{\prime}$ in $V$ are not endangered because of (c) and (d) and Lemma 1 (iv). (In case (d) obtains, an easy check of the construction shows that no element is listed in $H_{t}^{s}$ for any $t \geqq m^{\prime}$.)

Now let $h$ be such that

$$
\left\{\left(b_{i}=b_{j}\right)^{h(i, j)} \mid 1 \leqq i \leqq n+2\right\}
$$

is consistent with (\#), and let $\Sigma\left(x_{1}, \cdots, x_{n+2}\right)$ be the unique $(n+2)$ type of $T$ determined by the union of $(\#)$ and $\left({ }^{+\prime}\right)$, after the obvious substitution of variables for constants. By construction $\Sigma$ is e.z. Therefore so is the 2-type $\Phi\left(x_{n+1}, x_{n+2}\right)$ contained in $\Sigma\left(x_{1}, \cdots, x_{n+2}\right)$. Since $\Phi\left(x_{1}, x_{2}\right)$ is e.z., there must be a formula in $\Gamma\left(x_{1}, x_{2}\right)$ that is not in $\Phi\left(x_{1}, x_{2}\right)$. This formula is the desired $\gamma\left(x_{1}, x_{2}\right)$. Thus the lemma is proved, since it is straightforward to show that every e.z. type of $T$ has an index in $\left\{\hat{\Gamma}_{8} \mid s<\omega\right\}$.

By Lemma 4 , let $\mathscr{C}$ be a countable model of $T$ with witness set $\left\{\hat{\Gamma}_{s} \mid s<\omega\right\}$. Notice that the above omitting types argument can easily be amalgamated with the usual [1] proof that any countable model has a countable homogeneous elementary extension to show 
that $\mathscr{A}$ has a countable homogeneous elementary extension $\mathscr{C}$ which omits all types that are not e.z. Thus $\mathscr{C}$ is our desired countable homogeneous model with r.e. witness set $\left\{\hat{\Gamma}_{s} \mid s<\omega\right\}$. Therefore the theorem is finished with

LEMMA 5. No decidable model of $T$ realizes exactly the set of types $\left\{\Gamma_{s} \mid s<\omega\right\}$.

Proof. In order to obtain a contradiction, assume that $\mathscr{B}$ is a decidable model of $T$ realizing exactly the types $\left\{\Gamma_{s} \mid s<\omega\right\}$. Since $\mathscr{B}$ is decidable, the function $\mu: \omega \times \omega \times 2^{<\omega} \rightarrow 2$ such that

(1) $\mu(n, n, \underline{f})=0$ iff $\left(\mathscr{B}, \underline{b}_{n}\right) \vDash \Lambda_{i<l(\underline{f})} P_{i}\left(b_{n}\right)^{)^{f(i)}}$; and

(2) $\mu(n, m, \underline{g})=0$ iff $\left(\mathscr{B}, \underline{b}_{n}, \underline{b}_{m}\right) \vDash \Lambda_{i<l(\underline{g})} S_{i}\left(b_{n}, b_{m}\right)^{g(i)}$ for $n \neq m$ is recursive for some indexing $\left\{\underline{b}_{i} \mid i<\omega\right\}$ of $|\mathscr{B}|$. Fix an $m<\omega$ such that $\mu_{m}$ is such a $\mu$. It is easy to see that because $\square_{m}^{0}$ is first defined on an element in $F$ and $\mathscr{B}$ is a model of $T$, eventually in the construction of $F$ an $m$-fixed number $m_{0}$ appears. Fix an $r_{0}$ such that

$$
\left(\mathscr{B}, \underline{b}_{m_{0}}\right) \vDash P_{i}\left(b_{m_{0}}\right) \text { for all } i \geqq r_{0} .
$$

Such an $r_{0}$ exists, since $\mathscr{B}$ realizes only e.z. types. By the same argument there is an $m$-designated number $d$ at some stage $r_{0}^{\prime} \geqq r_{0}$ and an $s_{0} \geqq r_{0}^{\prime}$ such that

$$
\left(\mathscr{B}, \underline{b}_{d}\right) \vDash P_{i}\left(b_{d}\right) \text { for all } i \geqq s_{0} .
$$

Now, the only circumstance under which a number can cease being $m$-designated is the one covered in $(V)(C)$. Since (1\#) holds, it follows that $d$ is the $m$-designated number for all stages greater than $r_{0}^{\prime}$. But now, essentially by (IV) (C) and (1\#), (2\#) there is an $\left\langle\underline{f}_{0}, \underline{f}_{1}, \underline{g}\right\rangle \in H$ such that

$$
\left(\mathscr{B}, \underline{b}_{m_{0}}, \underline{b}_{d}\right) \vDash \Lambda_{i<l\left(\underline{f}_{0}\right)} P_{i}\left(b_{m_{0}}\right)^{\underline{f}_{0}(i)} \wedge \Lambda_{i<l\left(\underline{f}_{1}\right)} P_{i}\left(b_{d}\right)^{\underline{f}_{1}(i)} \wedge \Lambda_{i<l(\underline{g})} S_{i}\left(b_{m_{0}}, b_{d}\right)^{\underline{g(i)}} .
$$

But then $\mathscr{B}$ is not a model $T^{\prime}$, which is the desired contradiction.

It should be noted that $\left\{\Gamma_{s} \mid s<\omega\right\}$ also has a recursive witness set, this follows easily from the fact that each given partial recursive function has an infinite number of effectively recognizable indices. Therefore a strengthening along this line fails to produce a sufficient condition for ensuring decidability.

\section{REFERENCES}

1. C. C. Chang and H. S. Keisler, Model Theory, North-Holland Publishing Company, Amsterdam, 1973. 
2. L. Harrington, Recursively presentable prime models, J. Symbolic Logic, 39 (1974), 305-309.

3. T. S. Millar, Foundations of recurcive model theory, Annals of Math. Logic, 13 (1978), 45-72.

4. M. D. Morley, Decidable models, Israel J. Mathematics, 25 (1976), 329-340.

5. H. Rogers, Jr., Theory of Recursive Functions and Effective Computability, McGraw. Hill, New York, 1967.

Received May 22, 1978.

UNIVERSITY OF WISCONSIN

MADISON, WI 53706 


\section{PACIFIC JOURNAL OF MATHEMATICS}

\section{EDITORS}

DONALD BABBITT (Managing Editor)

University of California

Los Angeles, CA 90024

Hugo RossI

University of Utah

Salt Lake City, UT 84112

C. C. MOORE and ANDREW OGG

University of California

Berkeley, CA 94720

\section{J. DugundjI}

Department of Mathematics

University of Southern California

Los Angeles, CA 90007

R. FinN and J. Milgram

Stanford University

Stanford, CA 94305

\section{ASSOCIATE EDITORS}
E. F. BeCKENBACH
B. H. Neumann
F. WoLF
K. YoSHIDA

\section{SUPPORTING INSTITUTIONS}

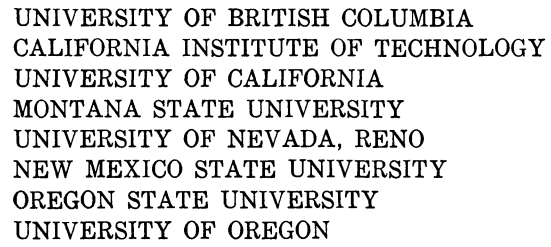

UNIVERSITY OF BRITISH COLUMBIA CALIFORNIA INSTITUTE OF TECHNOLOGY UNIVERSITY OF CALIFORNIA MONTANA STATE UNIVERSITY UNIVERSITY OF NEVADA, RENO NEW MEXICO STATE UNIVERSITY OREGON STATE UNIVERSITY UNIVERSITY OF OREGON

\author{
UNIVERSITY OF SOUTHERN CALIFORNIA \\ STANFORD UNIVERSITY \\ UNIVERSITY OF HAWAII \\ UNIVERSITY OF TOKYO \\ UNIVERSITY OF UTAH \\ WASHINGTON STATE UNIVERSITY \\ UNIVERSITY OF WASHINGTON
}

The Supporting Institutions listed above contribute to the cost of publication of this Journal, but they are not owners or publishers and have no responsibility for its content or policies.

Mathematical papers intended for publication in the Pacific Journal of Mathematics should be in typed form or offset-reproduced, (not dittoed), double spaced with large margins. Please do not use built up fractions in the text of the manuscript. However, you may use them in the displayed equations. Underline Greek letters in red, German in green, and script in blue. The first paragraph or two must be capable of being used separately as a synopsis of the entire paper. Please propose a heading for the odd numbered pages of less than 35 characters. Manuscripts, in triplicate, may be sent to any one of the editors. Please classify according to the scheme of Math. Reviews, Index to Vol. 39. Supply name and address of author to whom proofs should be sent. All other communications should be addressed to the managing editor, or Elaine Barth, University of California, Los Angeles, California, 90024.

50 reprints to each author are provided free for each article, only if page charges have been substantially paid. Additional copies may be obtained at cost in multiples of 50 .

The Pacific Journal of Mathematics is issued monthly as of January 1966. Regular subscription rate: $\$ 84.00$ a year (6 Vols., 12 issues). Special rate: $\$ 42.00$ a year to individual members of supporting institutions.

Subscriptions, orders for numbers issued in the last three calendar years, and changes of address shoud be sent to Pacific Journal of Mathematics, P.O. Box 969, Carmel Valley, CA 93924, U.S.A Old back numbers obtainable from Kraus Periodicals Co., Route 100, Millwood, NY 10546.

PUBLISHED BY PACIFIC JOURNAL OF MATHEMATICS, A NON-PROFIT CORPORATION

Printed at Kokusai Bunken Insatsusha (International Academic Printing Co., Ltd.). 8-8, 3-chome, Takadanobaba, Shinjuku-ku, Tokyo 160, Japan. 


\section{Pacific Journal of Mathematics}

\section{Vol. 91, No. 2 December, 1980}

Victor P. Camillo and Julius Martin Zelmanowitz, Dimension modules ... . . 249

Yonina S. Cooper, Stable sequences in pre-abelian categories ........... 263

Chandrakant Mahadeorao Deo and H. Ship-Fah Wong, On Berry-Esseen approximation and a functional LIL for a class of dependent random fields.........................................

H. P. Dikshit and S. N. Dubey, $|C, 1|$ summability of series associated with

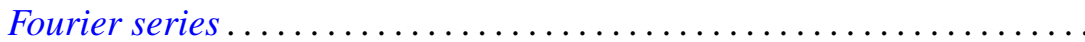

M. Edelstein, On the homomorphic and isomorphic embeddings of a semiflow into a radial flow.

Gilles Godefroy, Compacts de Rosenthal ..................... 293

James Guyker, Commuting hyponormal operators ................ 307

Thomas Eric Hall and Peter R. Jones, On the lattice of varieties of bands of

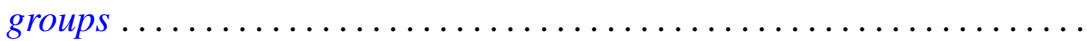

Taqdir Husain and Saleem H. Watson, Topological algebras with orthogonal Schauder bases ....................................

V. K. Jain, Some expansions involving basic hypergeometric functions of two variables. . .

Joe W. Jenkins, On group actions with nonzero fixed points ........... 363

Michael Ellsworth Mays, Groups of square-free order are scarce ........ 373

Michael John McAsey, Canonical models for invariant subspaces... 377

Peter A. McCoy, Singularities of solutions to linear second order elliptic partial differential equations with analytic coefficients by approximation methods...

Terrence Millar, Homogeneous models and decidability.

Stephen Carl Milne, A multiple series transformation of the very well poised

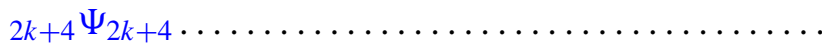

Robert Olin and James E. Thomson, Irreducible operators whose spectra are spectral sets...

Robert John Piacenza, Cohomology of diagrams and equivariant singular

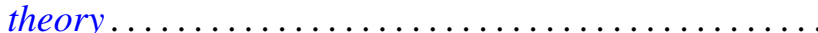

Louis Jackson Ratliff, Jr., Integrally closed ideals and asymptotic prime divisors

Robert Breckenridge Warfield, Jr., Cancellation of modules and groups and stable range of endomorphism rings.................

B. J. Day, Correction to: "Locale geometry" ...............

Stanley Stephen Page, Correction to: "Regular FPF rings" ... 